\title{
DESIGN WITH SADSF METHOD AND ANALYSES OF ELASTIC PROPERTIES OF TORSION-LOADED DOUBLE-TEE SECTION WITH TORSIONAL BOX
}

\author{
I. MARKIEWICZ \\ Faculty of Mechatronics and Mechanical Engineering \\ Kielce University of Technology \\ al. Tysiąclecia Państwa Polskiego 7, 25-314 Kielce, POLAND \\ E-mail: ireneusz.markiewicz@tu.kielce.pl
}

\begin{abstract}
The work presents the results of preliminary strength design of a thin-walled structure based on double-tee section loaded with a torsion moment. One of the solutions to this problem is considered, in which the torsional box is introduced in the central part. Then, one constructs a series of solution variants that differ in the torsional box length. In the design one uses the method of statically admissible discontinuous stress fields (SADSF) assuming the condition of equalized equivalent stress in the limit state.

The work is complemented with elastic FEM analyses of one of the solution variants. Using this example, one shows good load-carrying properties of structures designed with the SADSF method, and proves that they could be several times better than the properties of structures designed with traditional or intuitive ways.
\end{abstract}

Key words: design, thin-walled structures, limit analysis, computer methods.

\section{Introduction}

In this work, we present one of the solutions to the problem of preliminary strength design of a thinwalled structure. Formulation of the problem is illustrated in Fig.1a. The data are [1,2]: limit load $\boldsymbol{P}$ applied on the border $S_{p}$, reduced to two torsion moments: $M_{Y}=2 P h$, dimensions of this border $(L, h, e)$ and yield point of material of the designed structure $\left(\sigma_{y}\right)$.

One assumes that the sought after structure should be based on a double-tee section, contain a torsional box in its central part and exhibit shape symmetry, among other things, with respect to the plane denoted AS1 (Fig.1c), which is one of antisymmetry planes for external forces.

An additional element in this work is the construction and analysis of the solution variants that differ in the length of one half of the box, $t$ (Fig.1c).

The solution has been obtained with the use of the method of statically admissible discontinuous stress fields (SADSF [1-9]), which is based on the conclusions from the lower-bound theorem of limit analysis.

In the framework of this method, one looks for a statically admissible, discontinuous stress field, which is consequently equated with the designed structure.

Such a field satisfies the given boundary conditions, meets equilibrium equations at each point, and at no point does it disturb the assumed yield condition. In the SADSF method, one assumes that the sought after spatial field is built of fragments of plane fields, and the stress discontinuity lines are segments of straight lines, so they separate the areas of homogeneous stress state. When constructing the fields, one aspires to the fulfillment of the assumed yield condition in a possibly large number of areas.

The obtained field fully defines the designed construction, i.e., it defines both its structure, i.e., the number and spatial location of component elements and the system of their mutual connections [2], as well as geometry of these elements, i.e., their shapes and dimensions.

It is very difficult to undertake the task of designing and analyzing such fields in a direct way for a given system of boundary conditions. It leads to problems with nonlinear equations and inequalities that 
contain singularities and have large numbers of unknowns [3]. However, the most important thing is that, when the networks of division into homogeneous areas are assumed arbitrarily, the solutions might not exist [3]. Then, we deal with problems of unknown discretization, and the system of conditions that must be set up is not known, either.

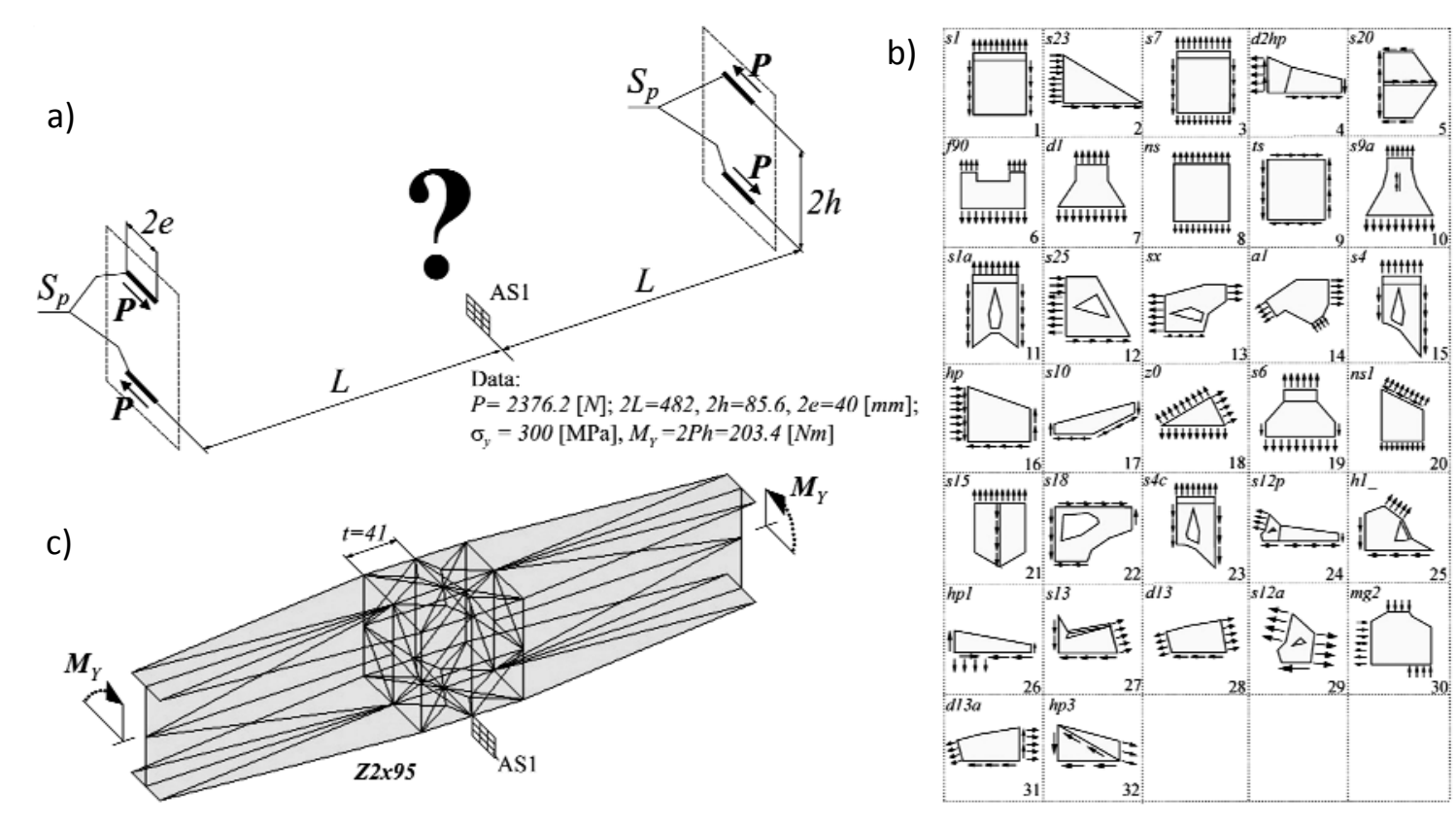

d)
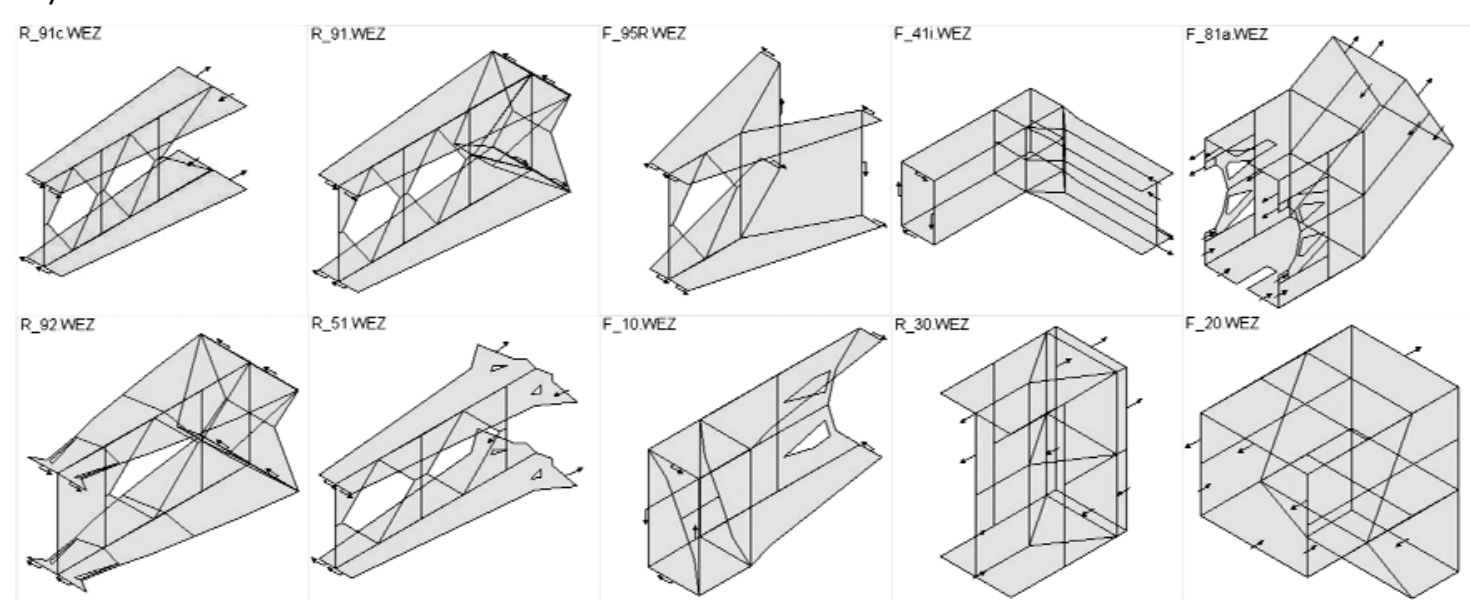

Fig.1. Formulation of design problem, one of solution variants and libraries of the package SADSFaM

a) formulation of design problem [1, 2]; b) ready-made library fields of the package SADSFaM [2] without stress discontinuity lines; c) one of variants of solution to the problem; statically admissible field obtained for $t=41[\mathrm{~mm}]$ with marked stress discontinuity lines; d) example fields from designer's library [2].

One managed to avoid these difficulties in the framework of the application version of the SADSF method [1, 2], which was created especially for engineers. It provides ready-made library fields (Fig.1b), which one may put together (like building blocks) creating more and more complicated systems.

In this work, we use the most advanced SADSFaM package of application version of the SADSF method, developed by W. Bodaszewski. It is based on the concept of multilevel approach to building complex fields [2], thanks to which one can simply and effectively design even very complicated thin-walled 
structures. In this concept, ready-made library fields provided with the software package, such as the ones depicted in Fig.1b, are classified as fields of level I. These are fields of low degree of complexity, satisfying the defined boundary conditions and are divided in a particular way into homogeneous areas (see Fig.2) for which the existence of solution has been confirmed.

Using the library fields, the designer can build more complex fields (of medium degree of complexity), which make the fields of level II. These fields, accommodated to the problems that the designer deals with, create the designer's library. The author of the package has already created several hundred of such fields, which satisfy diverse boundary conditions, and selected examples of which are presented in Fig.1d. The fields are consequently assembled to form the sought after solutions, which make the fields of level III and higher.

The effect of the assumed design criterion, which consists in the fulfillment of yield condition in a possibly large number of the areas, is that for the given boundary conditions one can sometimes find several solutions, and also - as it is shown in this work - to construct their variants that differ from one another in geometric parameters.

A series of such solution variants, obtained for different lengths of one half of the torsional box $t$, is presented in the following part of this work (Fig.4). One of the variants, obtained for $t=41[\mathrm{~mm}]$, is depicted in Fig.1c.

From among the obtained solution and their variants, one can select the cases that comply with some additional criteria. These may be, for example, associated with the lowest manufacturing costs, the lowest volume, or the best load-carrying properties.

As a certain 'fault' of the presented solution one can consider the fact that inner surfaces of the torsional box are not accessible from the outside, which can make it difficult to apply anti-corrosion protection. Examples of many other, interesting an original solutions to the problem from Fig.1a one can find in the monograph [2].

The work is complemented with the results of elastic FEM analyses of the solution variant shown in Fig.1c. Based on this example, we have shown good load-carrying properties of structures designed with the SADSF method. One could expect such properties, because they were confirmed by the analyses of several dozen of other thin-walled structures designed with the SADSF method $[10,11]$. In this work, it is shown that these properties can be even a dozen or more times better that those of structures designed in traditional or intuitive ways.

It is worth noticing that:

- problem formulation, similar to that presented in Fig.1a, is typically applied at the beginning of the design process, when one only knows the load carried by the construction, the yield point of material used to make it and functional requirements that the structure must fulfill;

- the SADSF method is aimed at designing thin-walled structures built of plane elements; this is a particular class of structures, for which even a small change in boundary conditions or structural parameters (construction details) may cause radical changes in load-carrying properties (see, i.e., [2]); for this class of structures, one must exercise great caution when applying design methods based on iterative improvements, such as the commonly-used topology optimization methods [12-14];

- in each library field, there is assumed realization of plane state of stress; thanks to this, the structures composed of such fields have the ability to carry the applied load with domination of the membrane state; this state is characterized e.g., by uniform distributions of stresses over the thickness of element walls, relatively low level of stresses and high rigidity; then, the designed constructions are well fit for the applied load and are free of cardinal errors, whose presence may cause that the forces would be carried with domination of the bending state and load-carrying ability would decrease several times; such errors are often encountered in constructions designed in traditional or intuitive ways;

- the effect of applying the SADSF method is that we obtain a completely designed construction $[1,2]$, and we know in advance that it will have good load-carrying properties [2, 4-11]; such constructions can be accepted at once, and in the case of high-performance ones we can analyze and improve them by means of the FEM; possible corrections should only apply to geometry and must be introduced outside the contours 
determined with the SADSF method, so as to avoid decreasing the assumed limit load-carrying capacity [6]; a change in structural parameters might radically worsen these capacities.

\section{Necessary information about SADSFaM package [2]}

In the framework of application version of the SADSF method, the designer uses ready-made library fields (Fig.1c) which he/she assembles like building blocks to form more and more complex systems (see, i.e., Figs 1c-d). To construct the solution presented in this work, we used only four types of library fields, these are presented in a magnified scale in Fig.2 with marked stress discontinuity lines.

As it is seen, each of the field types is denoted with a symbol (here: $H p, N s 1, s 1$ and $T s$ ) and is described in its own (local) system of coordinates $\left\{a_{1}, a_{2}\right\}$. Outer loads of the fields are defined by external stress parameters $h_{i}$, which are the coordinates of stresses applied on the borders, whereas location of these borders is described by external geometric parameters $z_{i}$, that have a character of linear dimensions.
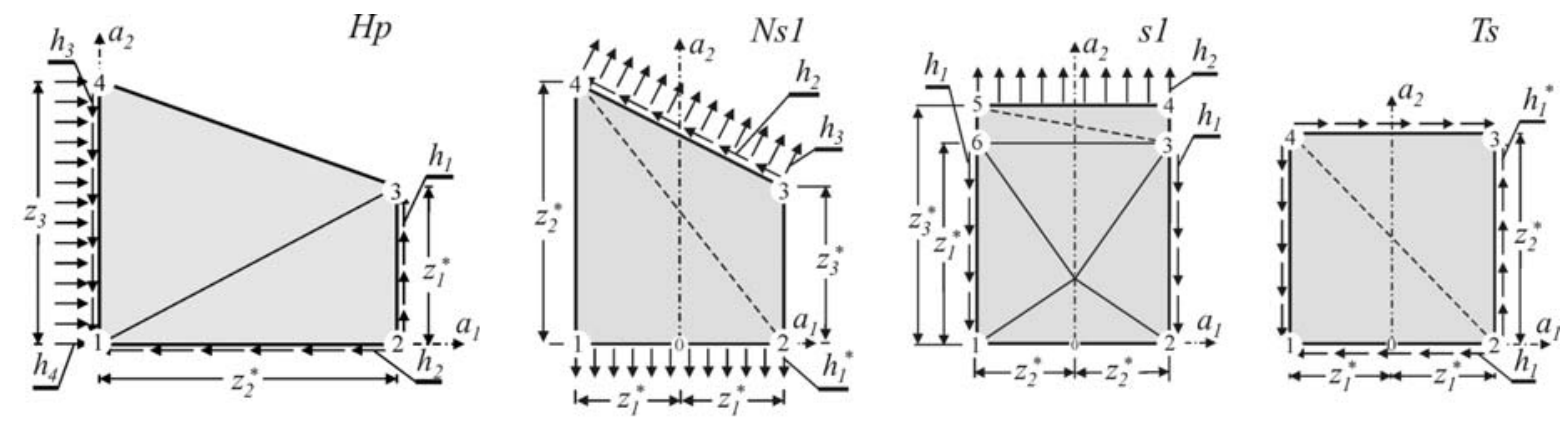

Fig.2. Library fields of SADSFaM package used in the presented solution.

Because the SADSFaM package makes use of the Treska yield condition, stress parameters on the borders of component fields are related to: $k=\sigma_{y} / 2=150[\mathrm{MPa}]$.

The fulfillment of equilibrium equations on each stress discontinuity line causes that global equilibrium equations of the field are satisfied identically. For example, for the field $s l$ (Fig.2c) we obtain one such equation, which takes the form: $z_{2} h_{2}=z_{1} h_{1}$. From what was mentioned it follows that not all external parameters $h_{i}$ and $z_{i}$ can be assumed independently. The parameters that are presupposed independent, the ones whose values are inputted in the package, are denoted with an asterisks in Fig.2.

A complex field, built of library fields, remains statically admissible if geometric and static condition of joining are satisfied. Geometric conditions require that the connected borders coincide, and static conditions consist in fulfillment of conditions of equality of mutual interactions on these borders.

In the cases of the simplest problems, solutions are constructed by directly connecting the library fields. However, this is a tedious and time-consuming task.

For this reason, in the cases of more complex problems, one first constructs fragments of solutions (e.g., fields of level II, see Fig.1d), which can consequently be multiplied and assembled to form ready solutions (fields of level III or higher).

In a complex field, each ready-made library field is given its own index $s$ (drawn in rectangular rims), and thickness of the element is denoted $\delta^{(s)}$.

\section{Construction of field in antisymmetric half of solution variant from Fig.1c}

In the solution presented in this work, we used two fields of level II. These fields, constructed for $t=41[\mathrm{~mm}]$, are shown in Figs $3 \mathrm{a}$ and $3 \mathrm{~b}$.

There, thin arrows denote resultant interactions between component fields, and thick arrows symbolize resultant external reactions.

The first of the fields, used for construction of the solution in the areas adjacent to the borders $S_{p}$ (Fig.1a) is denoted $A d \_95 a$ (the same as the name of the field's data file). To build the field, it was enough to 
find a solution for its antisymmetric half, which is presented in the upper part of Fig.3a. As we can see, it consists of two identical fields $H p$ that make up the flange, and the field $N_{s} l$ in the unloaded web. By assembling two such solutions, we have obtained the sought after field of level II, which defines the shape and dimensions of the flanges and the web of the double-tee section.

This solution is a version of the field denoted $R_{-} 91 c$ from Fig.1d [2]. In this solution, the number of fields $N_{s} 1$ in the web was reduced, so as to limit the amount of data introduced when constructing the solution variants for diverse values of $L-t$.

The second field of level II, which defines the shape and dimensions of the construction in one half of the torsional box, was denoted $A d$-95b. In this case, it was enough to solve only one antisymmetric quarter of it, which is shown in the upper part of Fig.3b. Here we applied four library fields: one field $s 1$ and three fields $T s$, which realize pure shear. The field of level II has been obtained by assembling four such solutions.

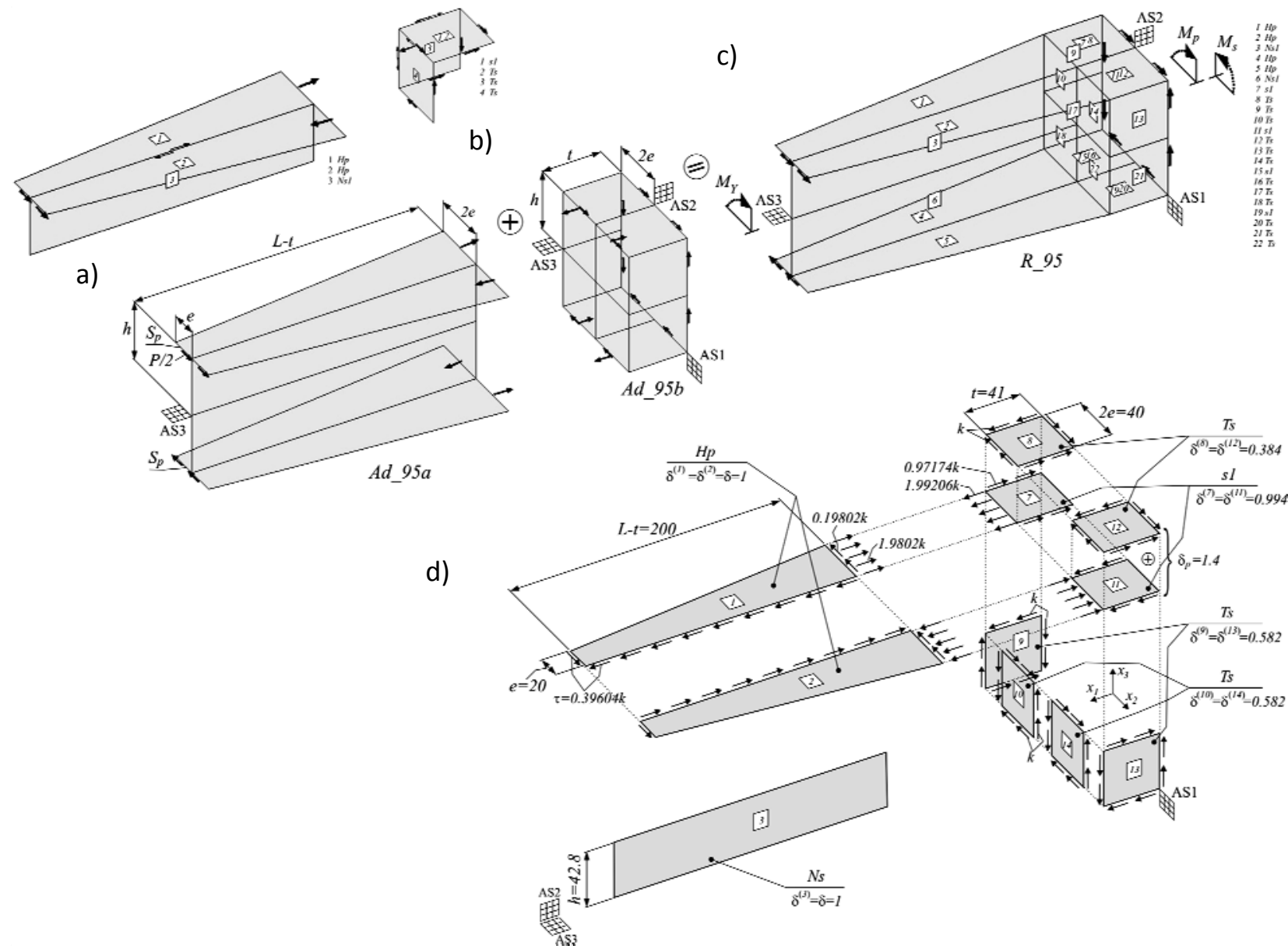

Fig.3. Field construction in antisymmetric half of solution to the problem from Fig.1a a) field $A d$ _95a that defines the shape and dimensions of web and flanges; b) field $A d \_95 b$ that defines the shape and dimensions of elements within torsional box; c) field $R_{-} 95$ that makes one half of solution to the problem from Fig.1a; d) location of component library fields, loads applied to fields and thicknesses of their elements in the upper half of field $R_{-} 95$.

Putting together the fields $A d \_95 a$ and $A d \_95 b$, as it is shown in Figs 3a and 3b, one obtains the solution $R_{-} 95$ depicted in Fig.3c, which constitutes one half of the solution to the problem formulated in Fig.1a and presented in Fig.1c.

The presented drawings are, in majority, screen dumps of images obtained from the SADSFaM package complemented with dimensioning and additional denotations; they are legible for the user of the package, but, among other things, they do not contain any numerical data. 
Because of that, details of the presented solution variant are shown in Fig.3d in a picture drawn with the use of a graphical editor. There are shown locations of component library fields in the upper half of the solution $R \_95$, arrows that symbolize loads and values of loads applied to fields, enumeration of component fields and their determined thicknesses. Interaction values and dimensions marked on the drawing may be easily associated with external parameters of the applied library fields from Fig.2.

Independent geometric parameters result from the given dimensions: $L, h, e$, the assumed dimension $t$, and geometric conditions of joining. Inputting these parameters and their correctness can be easily controlled on the display of field drawing on the monitor screen (transformation matrices that define spatial location of component fields are not discussed in this work).

It should also be added that, in the package SADSFaM, for the field $H p$ one assumes that: $z_{3}=2 z_{1}{ }^{*}$ (Fig.2a). It is so because, with the assumed length of the border $S_{p}$ equal to $2 e$, the width of the torsional box will in each case be the same, and be equal to $4 e$ (see Fig.3a).

It is worth noticing that the value of shear stress $\tau$ applied on the edges $H p$ adjacent to the border $S_{p}$, corresponds to the parameter $h_{l}$ of this field and is not assumed independently. When it is defined, then, after inputting independent parameters of fields $H p$, one can determine thickness of elements of these fields: $\delta^{(l)}=$ $\delta^{(2)}=\delta$, using the formula: $M_{Y}=2 P h=2(\tau 2 e \delta) h$.

Thicknesses of elements of the remaining fields are calculated from static conditions of joining. In the package SADSFaM, these conditions are automatically set up and solved. For example, for the common border between the fields $H p(s=1), s 1(s=7)$ and $T s(s=8,10)$ these conditions take the form

$$
\begin{aligned}
& (1.9802 k) \delta^{(1)}-(1.99206 k) \delta^{(7)}=0, \\
& (0.19802 k) \delta^{(1)}+(1.0 k) \delta^{(8)}-(1.0 k) \delta^{(10)}=0
\end{aligned}
$$

The total thickness of the flange within the torsional box is obtained summing thicknesses of elements of the fields $s l(s=7)$ and $T s(s=8): \delta_{p}=\delta^{(7)}+\delta^{(8)}$ (layer superimposition was not used $[1,8]$ ).

Only for the web, where the field type $N_{s} 1(s=3)$ and a zero state of stress is assumed, can one assume the element's thickness arbitrarily. It is accepted that its thickness is the same as that of flange elements, and equals $\delta^{(3)}=\delta$. The web's only role is 'to support' geometry, and because it is unloaded, one can - without any loss in load-carrying capacity - arbitrarily make holes in it.

It is worth analyzing here statically admissible forces, both the forces applied to component library fields and the resulting external reactions of fields of level II. As it is seen from Fig.3a, a bimoment appears, and its value rises as the distance from the edges adjacent to $S_{p}$ increases. Consequently, it is transmitted to the torsional box and 'locked' in it (Fig.3b). As we can see, in the cross-section AS1 (Fig.3c) the bimoment equals zero, because in this section there are not any perpendicular reactions which might create such a bimoment. In this section, forces from the flanges produce a torsion moment $M_{p}$, while the forces acting on the webs (perpendicular elements of the torsional box) create the moment $M_{s}$.

The flanges are bent in their planes. So, in the elastic range, axes of bending will appear in these elements, and the stresses will increase with increasing distance from the axes. One can expect that the elastic equivalent stress will only be equalized along the free borders, whereas uniform distributions of elastic equivalent stress can be expected in elements of the torsional box, where the state of pure shear is assumed.

\section{Selected solution variants}

The analysis of domains of independent external parameters of used library fields has shown that the solution to the problem under consideration can be built for the dimension $t$ changing within very large limits. In this work, the solution was found in the range: $20 \leq t \leq 240[\mathrm{~mm}]$.

Selected examples of solution variants obtained for: $t=30,120,175$ and $239[\mathrm{~mm}]$ are presented in Figs 6a-d. For each field, there are shown external loads, thicknesses of field elements and the total volume of the field.

It is worth directing our attention to external reactions of these fields in the planes denoted AS1 in Figs 4a-d. Senses of reactions on the flange edges are, for $t<120$, the same as those of forces applied to the 
border $S_{p}$ (see Figs $3 \mathrm{c}$ and $4 \mathrm{a}$ ). For $t \approx 120$ these forces take zero values (Fig.4b) - which is due to the obtained zero thickness of the field elements $T_{s}(s=8,12)$ from which these forces result (see Fig.3d). For $t>120$ the forces take opposite senses, similarly as the senses of stresses in the fields $T s$. As it is shown in Fig.4e, the values of moments: $M_{p}$ and $M_{s}$ (see Fig.3) grow very quickly when the length of one half of the torsional box decreases. For $t=20[\mathrm{~mm}]$ they are 5-6 times greater than the loading moment $M_{Y}$. The most advantageous values of these moments take for $t$ values close to $L$.

a)

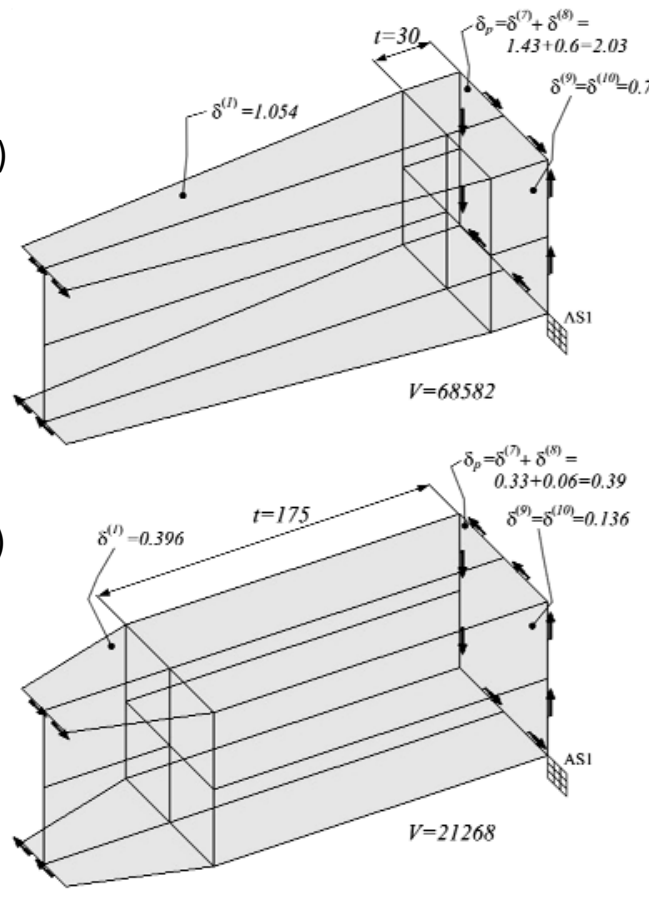

b)
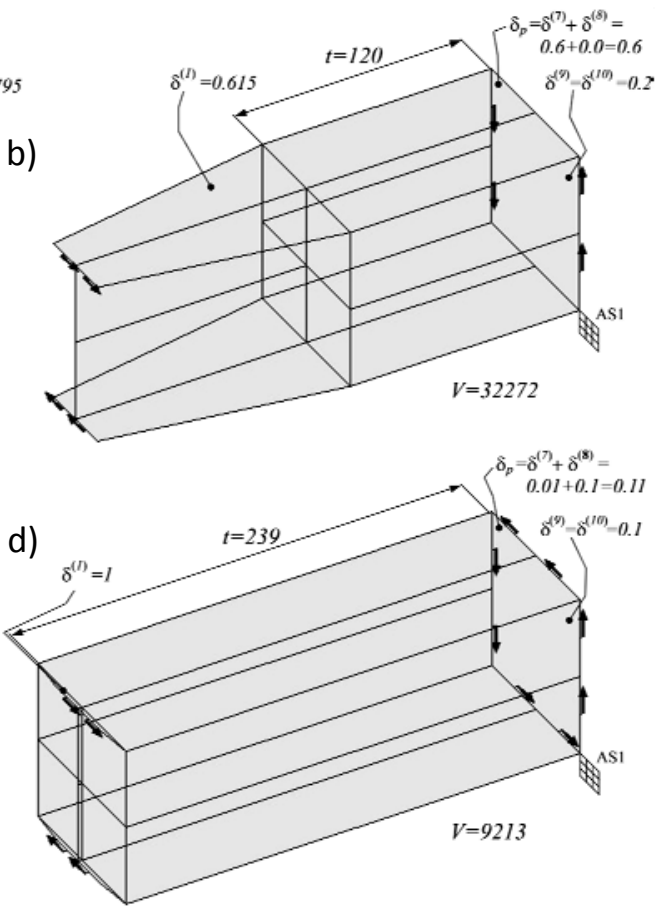

e)

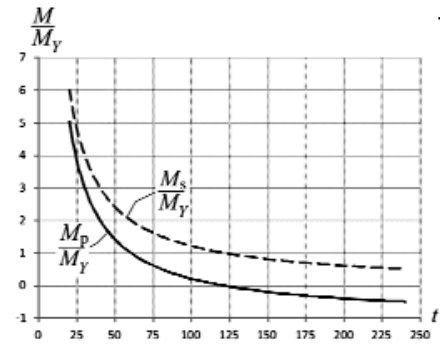

f) $8[\mathrm{~mm}]$

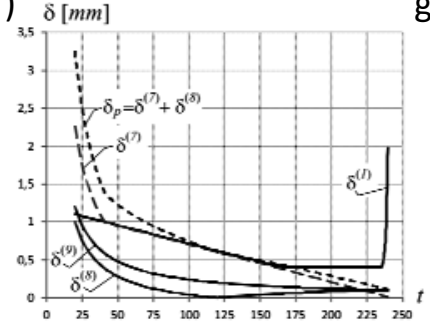

g)

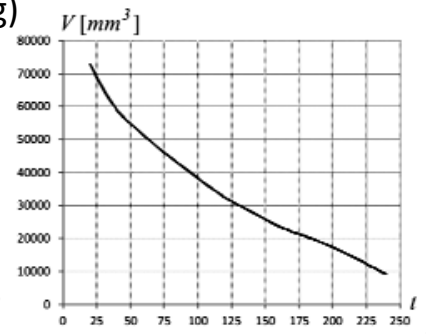

Fig.4. Example solution variants and results obtained. a, b, c, d) solution variants obtained for selected values of dimension $t$; e) courses of moments $M_{p}$ and $M_{s}$ referred to $M_{Y}$, in the function of dimension $t$; f) obtained thicknesses of component field elements versus dimension $t$; g) field volume versus dimension $t$.

We have also analyzed thicknesses of component fields. It is worth standardizing them, in order to lower manufacturing costs. The calculated courses of thicknesses versus dimension $t$ are presented in Fig.4f. As one can see, for $t<40$ the flange thickness $\delta_{p}$ in the torsional box area grows rapidly, whereas for $t>235$ the thickness of flanges outside the torsional box $\delta^{(I)}$ exhibits a rapid growth. In the range $100<t<175$, the thicknesses of elements $\delta^{(I)}$ and $\delta_{p}$ are very close to one another. Then, we can assume that these elements have the same thickness, equal to the greater of the two.

The thicknesses of elements that compose the torsional box are equal: $\delta^{(9)}=\delta^{(10)}$ and decrease with growing $t$. When $t$ approaches $L$, the thickness of these elements tends to that of the flanges in the torsional box area, $\delta_{p}$. 
The function of field volume $V$ versus $t$, which also represents the weight of the construction, is presented in Fig.6g. As it is seen, this volume decreases with the increase in length of one half of the torsional box, $t$.

The increase in dimension $t$ causes a decrease in the length of the fields $H p$ in the flanges, which are bent in their planes. So, the value of bimoment introduced to the torsional box decreases. Yet, the dimensions of the fields $T s$ that realize pure shear increase, and the solution tends to a rectangular, torsional pipe of equal thicknesses of all component elements. The presence of the fields $H p$ causes that the torsional box width is twice greater than the border length $S_{p}$. If these fields are removed from the solution, we obtain the solution presented in Fig.5a. It consists of eight fields $T s$, which have elements of equal thickness, and form a rectangular pipe closed with the fields $T_{s}(s=4,8)$. The locations of library component fields in the upper half of this solution, arrows that symbolize loads and values of loads applied to the fields, enumeration and the determined thickness values of component fields are presented in Fig.5b.
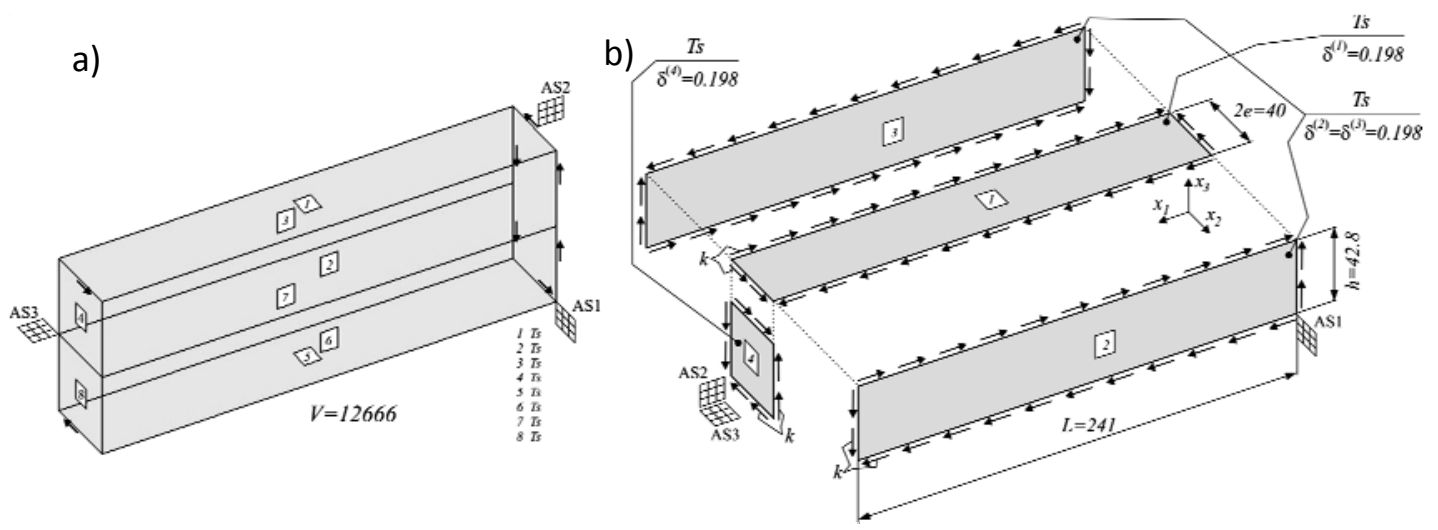

Fig.5. One half of the solution to the problem from Fig.1a in the form of torsional, rectangular pipe.

\section{Selected results of elastic FEM analyses}

Experimental and numerical verification of properties of constructions designed with the SADSF method has been carried out since the very beginning of existence of this method, because the method is an approximate one. In it, one makes use, among other things, of a rigid-ideally plastic material model, analyses only limit state of the structure corresponding to the beginning of its collapse, and assumes that, when the collapse occurs, a plane state of stress is maintained in each component element. Then, the elastic and elastic-plastic ranges are not taken into account, and bending states and phenomena associated with the loss of stability, etc., are not considered either.

However, the results obtained so far indicate surprisingly good load-carrying properties of the designed constructions in all stages of loading [2, 4-11], including the elastic range, in which the constructions usually work.

These properties have also been confirmed by the results of FEM analyses carried out by the author on numerous examples described, e.g., in the work [11]. In the SADSF-designed thin-walled structures, we have found, among other things, domination of membrane state, relatively well equalized level of equivalent stress - both along free borders and in large fragments the volume - and load-carrying properties radically better than such properties of systems whose structures are not properly designed for the applied load.

The results obtained for the presented solution variants confirm the above-mentioned good load-carrying properties. Then, in this work, we limited the presentation of results to just one case of the solution from Fig.1c. The results are shown together with those obtained for a model based on a regular, double-tee section, which was not designed with the SADSF method, and whose structure is not fit for torsional moment load.

The comparison of results obtained for the two models allowed us to show, among other things, what could be the advantage of applying the SADSF method to the design of thin-walled structures. 
In the analyses, we have used the CosmosM package. We have applied a shell model and triangular elements type SHELL6 with six nodes and six degrees of freedom in a node. We have also assumed, among other things, small displacements and strains and linearly-elastic model of the material.

The shapes and dimensions of the analyzed models, as well as the assumed boundary conditions, are presented in Figs $6 \mathrm{a}$ and $6 \mathrm{~b}$. As it is seen, we considered only quarters of the constructions, assuming adequate geometric boundary conditions in the antisymmetry planes denoted AS1 and AS3. The torsional moment was introduced in the membranes $p 1$ and $p 2$ by means of forces applied to the nodes lying on the edges of holes.

In the SADSF model (Fig.6a), we introduced some obvious corrections to geometry, which consisted in: increasing widths of the flanges by $5[\mathrm{~mm}]$ beyond the torsional box, assuming constant flange thickness equal to $1.4[\mathrm{~mm}]$ (see Fig.3d), and making holes in the unloaded web. The load value $F$ was assumed so as to obtain a moment equal to one half of the value assumed in the design.

a)

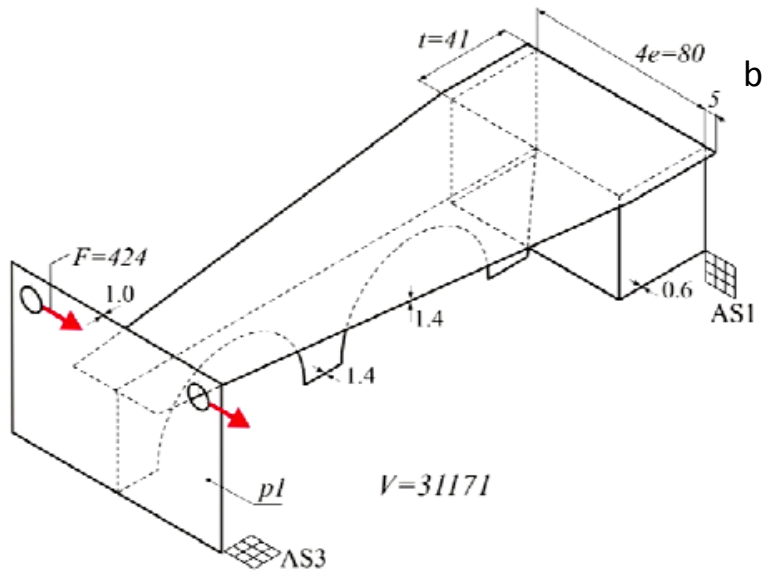

c)

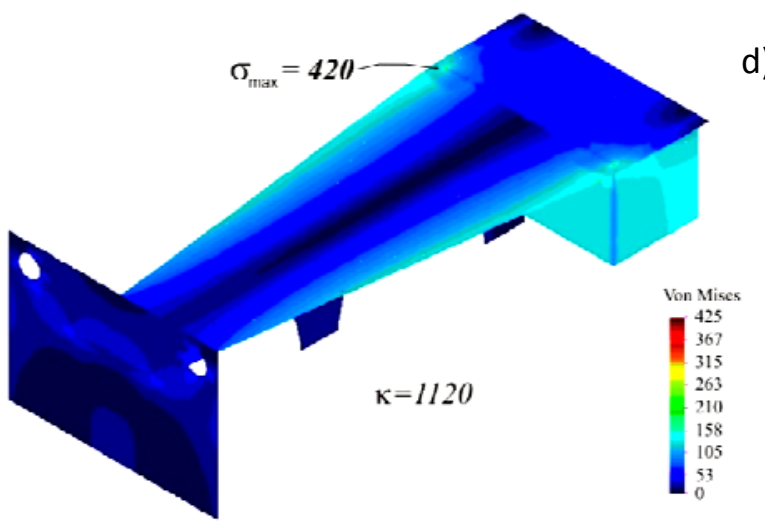

b)

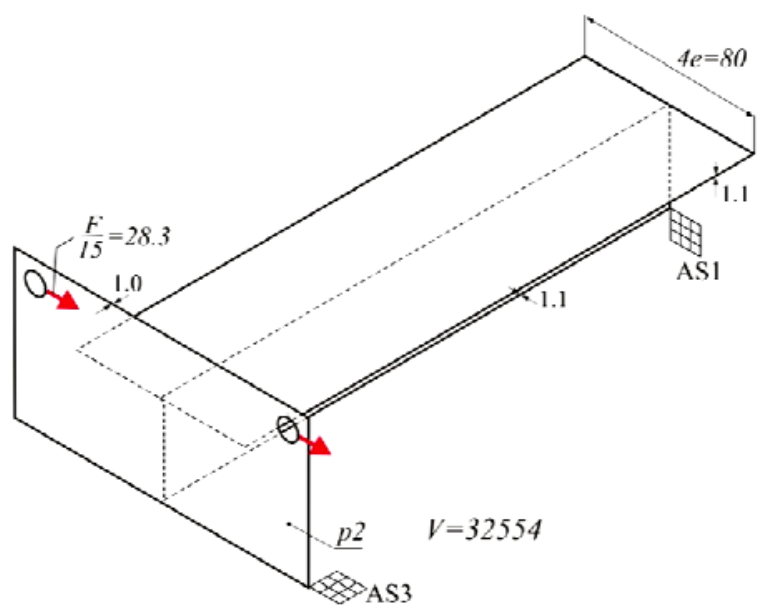

d)

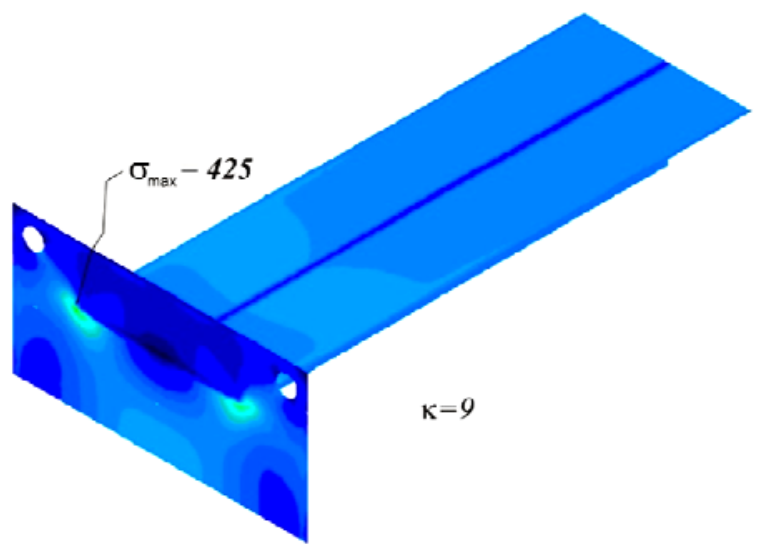

Fig.6. Assumed shapes, dimensions and boundary conditions of analyzed models, and obtained distributions of equivalent stresses calculated based on the Huber-Mises criterion.

The distribution of equivalent stress, obtained for this model, is shown in Fig.6c, where one can see:

- relatively well equalized equivalent stress along free borders of the flanges, and small stresses in their central part (in the torsional box area);

- very good level of equalization of equivalent stress in the elements of the torsional box, where pure shear was assumed in the statically admissible field;

- absence of load in the web, in which a zero state of stress was assumed.

We have found domination of the membrane state - the greatest equivalent stress related to the membrane state is almost twice greater than the maximal stress of bending state $(352 / 188 \approx 1.9)$. 
Rigidity of this model equals: $\kappa=M / \phi \approx 1120[\mathrm{Nm} / \mathrm{deg}]$; where $M$ - value of applied torsional moment, $\phi$ - rotation angle of upper edge of membrane $p l$ calculated based on displacement values of its extreme nodes.

In the case of the double-tee section model, we assumed constant width of the flanges equal to the torsional box width. To ensure that the amount of material is approximately the same as in the SADSF model, we assumed the same thickness for all elements in this model, equal to $1.1[\mathrm{~mm}]$ (Fig.6b). In order to make the maximum value of total equivalent stress equal to that in the SADSF model, we had to decrease the load value $F$ as much as 15 times.

Such a great difference in the applied load values results from the fact that the structure designed with the SADSF method carries the applied load with domination of the membrane state. Such a possibility does not exist in the regular double-tee section. In this case, the bending state is the dominating one - the greatest equivalent stress originating from bending state is more than twice greater than that obtained for the membrane state $(309 / 138 \approx 2.24)$.

The rigidity of the regular double-tee section model equals: $\kappa \approx 9[\mathrm{Nm} / \mathrm{deg}]$ and is about 124 times $(1120 / 9 \approx 124)$ smaller than that of the model designed with the SADSF method.

\section{Summary}

In this work, we have shown the simplicity and effectiveness of design of thin-walled structures by means of the application version of the SADSF method, and demonstrated easiness of constructing their variants. The method has the potential to find wide application in practice, already in the phase when only boundary conditions are known.

The package SADSFaM, applied in this work, is user-friendly and has no limitation on the complexity of designed systems. To obtain the solution, it is enough that the designer shows inventiveness in selecting and assembling the library fields, and is familiar with the concept of equilibrium.

The condition of equalized equivalent stress, assumed in the SADSF method, is ambiguous, and sometimes allows us to construct several solutions for the given boundary conditions [1, 2], as well as finding solution variants differing in geometric parameters. From among them, we can select the ones which meet some additional criteria, associated, e.g., with manufacturing costs or strength. The additional criteria can be taken into account already at the stage of selecting and assembling library fields, which is very useful in practical applications [2].

Preliminary projects of constructions, obtained with the use of the SADSF method, have the structures fit for the applied loads, and are free of cardinal errors. Therefore, their load-carrying properties might be radically better than the properties of systems designed with traditional methods. The systems designed with the SADSF method can, in most cases, be accepted at once. Only in a few cases they need improvements in geometry.

\section{Nomenclature}

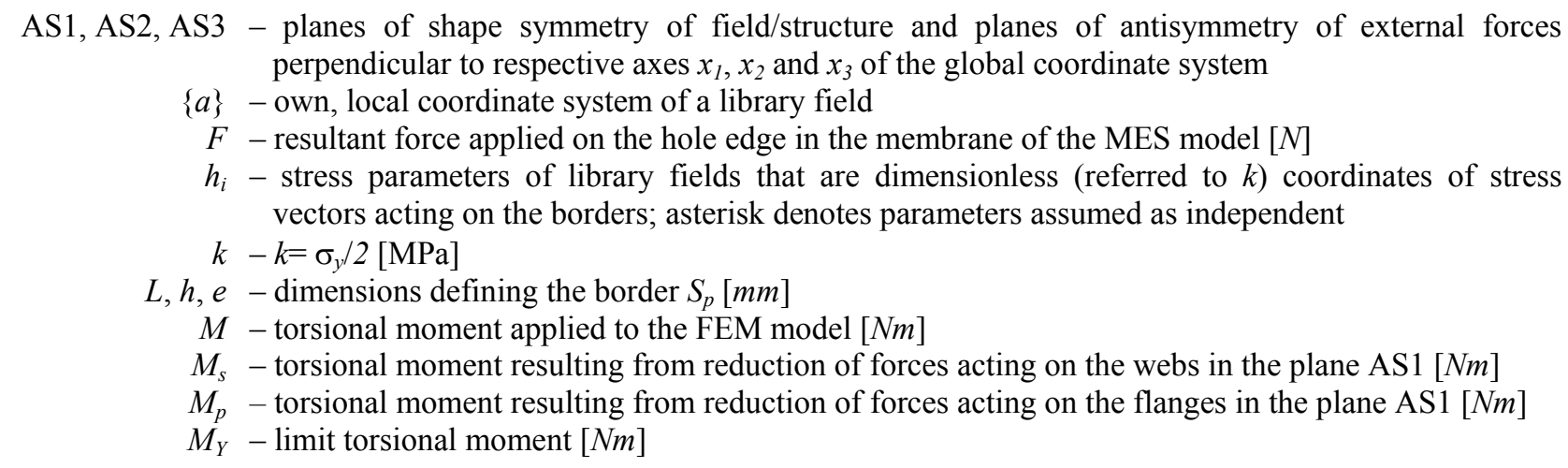




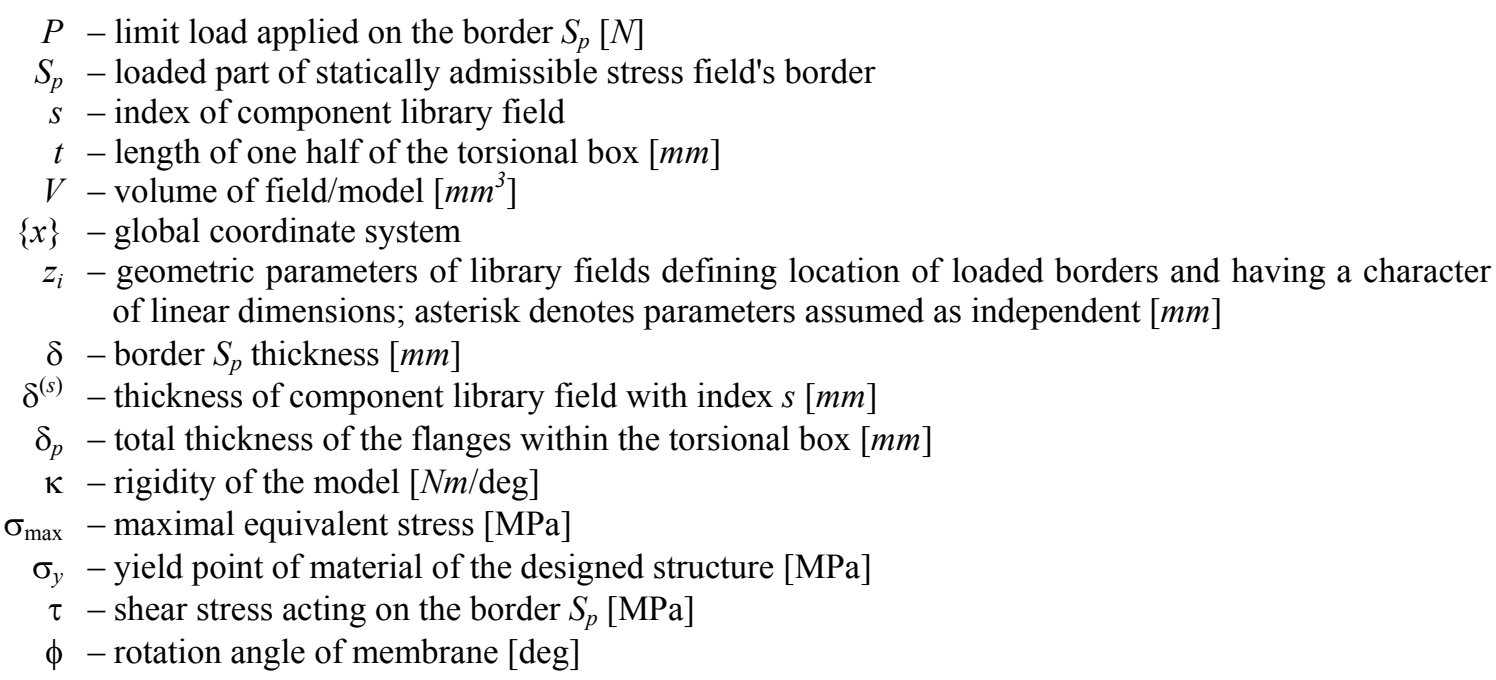

\section{References}

[1] Bodaszewski W. and Szczepiński W. (2005): Shaping structure elements by the method of discontinuous stress fields. - Warsaw: BEL Studio, PWN (in Polish).

[2] Bodaszewski W. (2013): Statical analyses and shaping of complex thin-walled structures. - Warsaw: BEL Studio (in Polish).

[3] Bodaszewski W. (2004, 2005): Algorithms of the method of statically admissible discontinuous stress fields $(S A D S F)$. - Engineering Transactions, vol.52, No.3, pp.175-193; vol.52, No.4, pp.281-302; vol.53, No.1, pp.1530; vol.53, No.2, pp.119-131.

[4] Dietrich L., Miastkowski J. and Szczepiński W. (1970): Load-carrying capacity of structural elements. - Warsaw: PWN (in Polish).

[5] Frąckiewicz H. et al. (1985): Joints and Structural Connections. - Warsaw: WNT (in Polish).

[6] Szczepiński W. (1968): Plastic Design of Machine Parts. - Warsaw: PWN (in Polish).

[7] Szczepiński W. and Szlagowski J. (1990): Plastic design of complex shape structures. - Warsaw - Chichester: Ellis Horwood and PWN.

[8] Szlagowski J. (1990): Methodology of strength design of structural elements according to the limit load carrying capacity criterion. - IFTR Reports, 25 (in Polish).

[9] Zowczak W. (2004): Strength design by means of slip-line method. - MONOGRAFIE, STUDIA, ROZPRAWY M-41, Wydawnictwo Politechniki Świętokrzyskiej, Kielce (in Polish).

[10] Markiewicz I. (2007): Analysis of elastic effort fields in truck frame designed by the SADSF method. Eksploatacja i Niezawodność - Maintenance and Reliability, vol.2, No.34, pp.22-27.

[11] Markiewicz I. (2013): Investigating the behaviour of structures designed with the SADSF method. MONOGRAFIE, STUDIA, ROZPRAWY M47, Wydawnictwo Politechniki Świętokrzyskiej, Kielce (in Polish).

[12] Bendsoe M.P. and Sigmund O. (2003): Topology Optimization: Theory, Methods, and Applications. - Springer.

[13] Huang X. and Xie Y.M. (2010): Evolutionary Topology Optimization of Continuum Structures. Methods and Applications. - Willey.

[14] Mróz Z. and Bojczuk D. (2003): Finite topology variations in optimal design of structures. - Structural and Multidisciplinary Optimization, vol.25, No.3, pp.153-173.

Received: May 10, 2018

Revised: November 13, 2018 